\title{
A Case of Ruptured Blood Blister-like Aneurysm Treated with Pipeline Embolization Device: Clinical Significance of Fetal-type Posterior Communicating Artery
}

\author{
Ki-Su Park, MD', Dong-Hun Kang, MD',3, Won-Soo Son, MD², Jaechan Park, MD, PhD'2, \\ Young-Sun Kim, MD, PhD³, Byung Moon Kim, MD, PhD ${ }^{4}$
}

Blood-blister like aneurysms (BBAs) are challenging lesions because of their wide fragile neck. Flowdiverting stents (FDSs), such as the Pipeline Embolization Device (PED), have been applied to treat BBAs less amenable to more established techniques of treatment. However, the use of FDSs, including the PED, in acute subarachnoid hemorrhage (SAH) still remains controversial. We report a case of aneurysm regrowth following PED application for a ruptured BBA that overlapped the origin of the dominant posterior communicating artery (PCOA), which was successfully treated after coil trapping of the origin of the fetal-type PCoA. And, we discuss the clinical significance of the fetal-type PCoA communicating with a BBA in terms of PED failure.

Key Words : Blood blister-like aneurysm; Flow diversion; Pipeline Embolization Device; Fetal circulation; Coil embolization

\footnotetext{
'Department of Neurosurgery, Chung-Ang University College of Medicine, Seoul, Korea

${ }^{2}$ Department of Neurosurgery, Kyungpook National University School of Medicine, Daegu, Korea

${ }^{3}$ Department of Radiology, Kyungpook National University School of Medicine, Daegu, Korea

${ }^{4}$ Department of Radiology, Younsei University College of Medicine, Seoul, South, Korea

Received October 6, 2016;

accepted after revision January 2, 2017.

Correspondence to: Dong-Hun Kang, MD, Department of Neurosurgery and Radiology, Kyungpook National University School of Medicine, 101 Dongin-dong $2 \mathrm{Ga}$, Jung-gu, Daegu 41944, Korea.

Tel. 82.10.9611.9461 Fax. 82.53.423.0504

E-mail: kdhdock@hotmail.com

This is an Open Access article distributed under the terms of the Creative Commons Attribution Non-Commercial License (http://creativecommons.org/licenses/by-nc/3.0) which permits unrestricted non-commercial use, distribution, and reproduction in any medium, provided the original work is properly cited.
}

Blood-blister like aneurysms (BBAs), which usually arise from the superior or superomedial wall of the supraclinoid internal carotid artery (ICA), are thinwalled and broad-based aneurysms that lack an identifiable neck [1]. Because of their fragile character, BBAs remain a challenge to treat safely, regardless of the surgical or endovascular treatment employed.

Recently, flow-diverting stents (FDSs), such as the Pipeline Embolization Device (PED), have been applied to treat ruptured BBAs with subarachnoid hemorrhage $(\mathrm{SAH})$, generally with positive results [2]. Nevertheless, enlargement and fatal re-rupture have been reported in some cases in spite of successful PED placement to treat the ruptured BBA [3]. A possible cause of regrowth or re-rupture in these cases is that a BBA following PED placement is not immediately 


\section{Ruptured BBA treated with Pipeline Embolization Device}

excluded from circulation, as opposed to microsurgical clipping or direct coil embolization. Therefore, the use of a PED in cases of a ruptured BBA remains controversial.

We report a case of aneurysm regrowth following PED application for a ruptured BBA. We concluded the BBA overlapped the origin of the fetal-type posterior communicating artery (PCoA), potentially allowing regrowth via continuous blood flow from the ICA through BBA to PCoA, which in turn alleviated the flow diversion effect. We thereby performed coil trapping of the origin of the PCoA and achieved successful flow diversion.

\section{CASE REPORT}

\section{History and Examination}

A 52-year-old female with no history of disease presented with a sudden bursting headache and transient loss of consciousness. She was admitted to other local hospital. An immediate CT scan showed a diffuse, thick SAH in the basal cisterns and bilateral sylvian fissures (Fig. 1A). Subsequent digital subtraction angiography (DSA) revealed a small hemispheric bulge at the dorsomedial wall of the communicating segment ICA, which strongly suggested a ruptured BBA due to its location and morphology (Fig. 1B). At that institute, three self-expandable stents (LVIS, MicroVention, Aliso Viejo, CA, USA) were deployed into communicating segment of the ICA for flow diversion, and dual antiplatelet medication was administered immediately after the procedure (Fig. 1C and D). However, follow-up DSA at 5 days revealed regrowth of the BBA ( $>5 \mathrm{~mm})$, and the patient was referred to our institute (Fig. 1E).

\section{Operation}

In terms of treatment modality, surgical treatments were considered initially, but there existed a high probability of intraoperative morbidity because of the
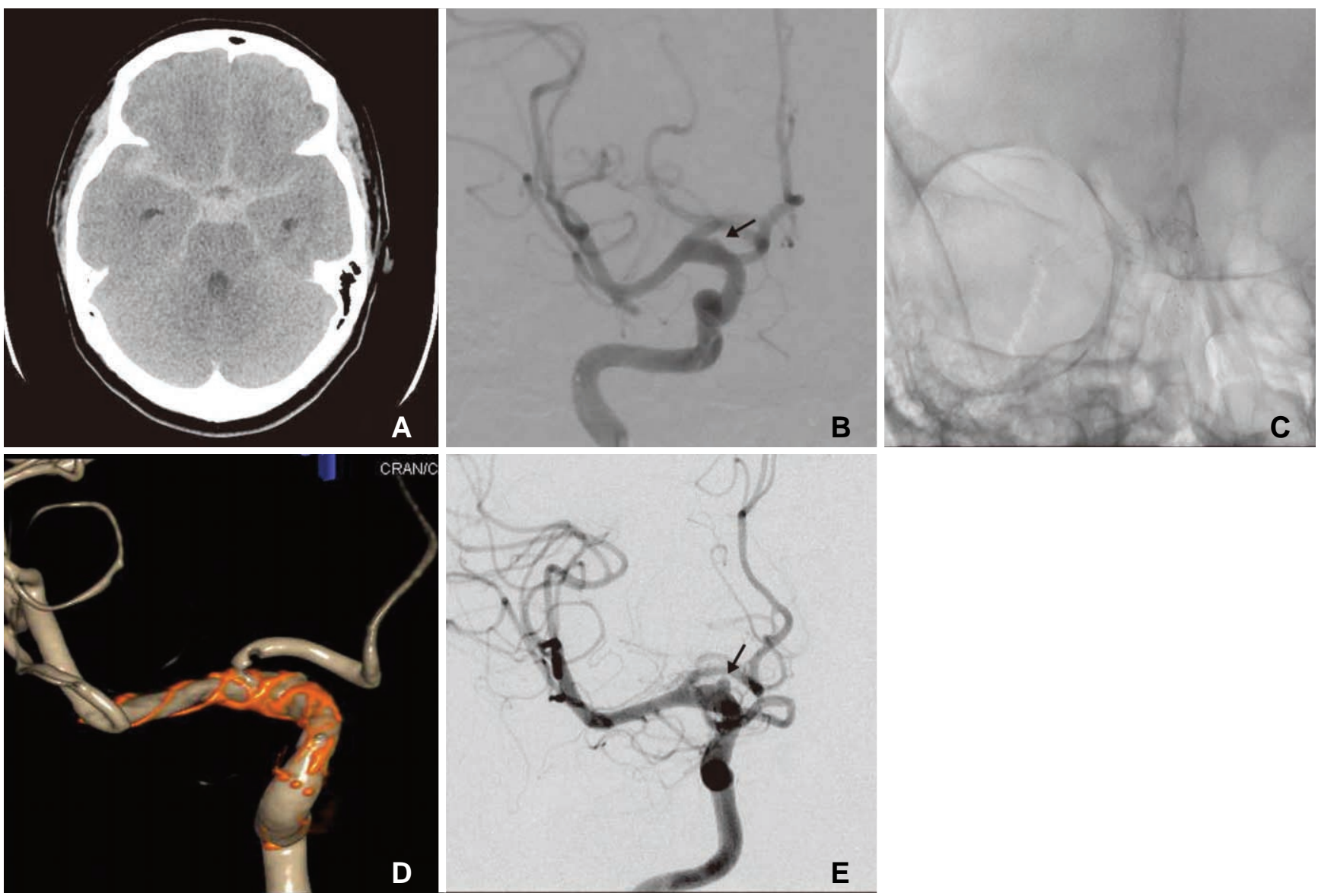

Fig. 1. Studies and an endovascular treatment at other local hospital (A) An immediate CT scan showed a diffuse, thick subarachnoid hemorrhage in the basal cisterns and bilateral sylvian fissures. (B) DSA revealed a small hemispheric bulge at the dorsomedial wall of the communicating segment ICA (arrow). (C and D) Three self-expandable stents (LVIS) were deployed into communicating segment of the ICA. (E) Follow-up DSA at 5 days demonstrated regrowth of the BBA (arrow). 
dual antiplatelet medication, anterior choroidal artery origin very closed to the BBA, and multiple overlapped stents. Subsequently, the implantation of a PED (Covidien-Ev3, Irvine, CA, USA) was attempted. At the end of procedure, DSA showed complete flow diversion and no contrast filling into the BBA (Fig. 2). However, follow-up DSA 10days after the first PED deployment revealed intraaneurysmal contrast filling and regrowth (Fig. 3A). This could be possibly explained by three hypotheses: 1) incomplete wall apposition of the first PED, 2) insufficient flow diversion using a single PED, and 3 ) inflow from the ICA through BBA to the fetal-type PCoA, which was connected to the neck of the BBA. Based on the aforementioned hypotheses, additional treatments were planned in the following order and immediately attempted. Firstly, balloon angioplasty within the first PED was performed to achieve complete apposition to the ICA wall (Fig. 3B). Then, one more PED was implanted within the previous PED for stronger flow diversion (Fig. 3C). However, contrast filling into the BBA still remained after the procedures (Fig. 3D and E). Eventually, coil trapping at the origin of the fetaltype PCoA was planned. A preoperative Allcock test showed retrograde filling of the fetal-type PCoA through the ipsilateral P1 segment. Under a 5-F guiding catheter (Envoy, Cordis) within the left vertebral artery, single microcatheter (Excelsior ${ }^{\circledR}$ SL-10 ${ }^{\circledR}$ pre 45 ) was advanced to the origin of the PCoA, then a total of 5 coils (Target ${ }^{\circledR} 360$ ultra $3 \times 6,3 \times 6,3 \times 4,2 \times 3$, and Target $^{\circledR}$ nano $^{\mathrm{TM}} 1.5 \times 3$ ) were deployed (Fig. 3F, G, and $\mathrm{H})$. Finally, the following DSA showed complete occlusion of the origin of the PCoA and no contrast filling into the BBA from ICA (Fig. 3I, J, K, and L).

\section{Postoperative course}

The postoperative clinical course was uneventful. Follow-up DSA was performed at 2 weeks, 1, and 3 month, which showed no contrast filling into the BBA, along with retrograding filling of the PCoA from the ipsilateral PCA. The patient was discharged without any neurological deficit.

\section{DISCUSSION}

FDSs are new, important tools in the treatment of intracranial aneurysms that redirect blood flow to promote stasis and delayed thrombosis within the aneurysm and reconstruction of the parent artery [4]. There are several reports of FDS use after SAH, typically in aneurysms less amenable to more established techniques of treatment, such as BBAs and fusiform aneurysms $[3,5,6]$. However, the use of FDSs, including the PED, in acute SAH still remains controversial due to several potential concerns.

The potential drawback is the chance of regrowth and rebleeding of aneurysms after FDS placement. Possible explanations in the literature have included the "hemodynamic theory", based on computational hemodynamic analysis, which is related both to the inherent flow diversion into a higher resistance parent artery pathway in combination with cerebral autoregulation, leading to higher pressure gradients and to changes in the parent artery configuration, such as reduction of proximal parent artery stenosis. Eventually, the intraaneurysmal pressure can be
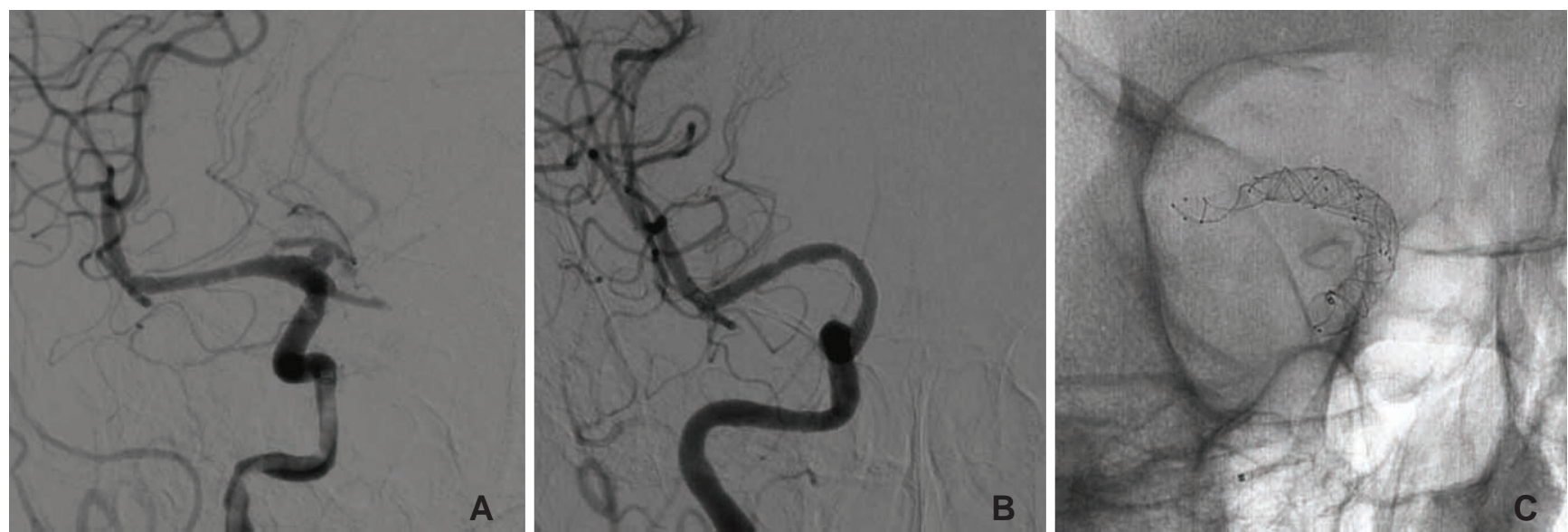

Fig. 2. The first PED implantation procedure for the BBA (A) Baseline angiography in our institute showed more prominent growth of the BBA and some amount of in-stent thrombosis. (B and C) Subtracted and non-subtracted angiographies after the first PED implantation, which demonstrated complete flow diversion of the BBA but also showed occlusions of ipsilateral proximal ACA and PCoA. The following left ICA and VA angiographies showed sufficient collateral flow through anterior and posterior communicating arteries. 


\section{Ruptured BBA treated with Pipeline Embolization Device}
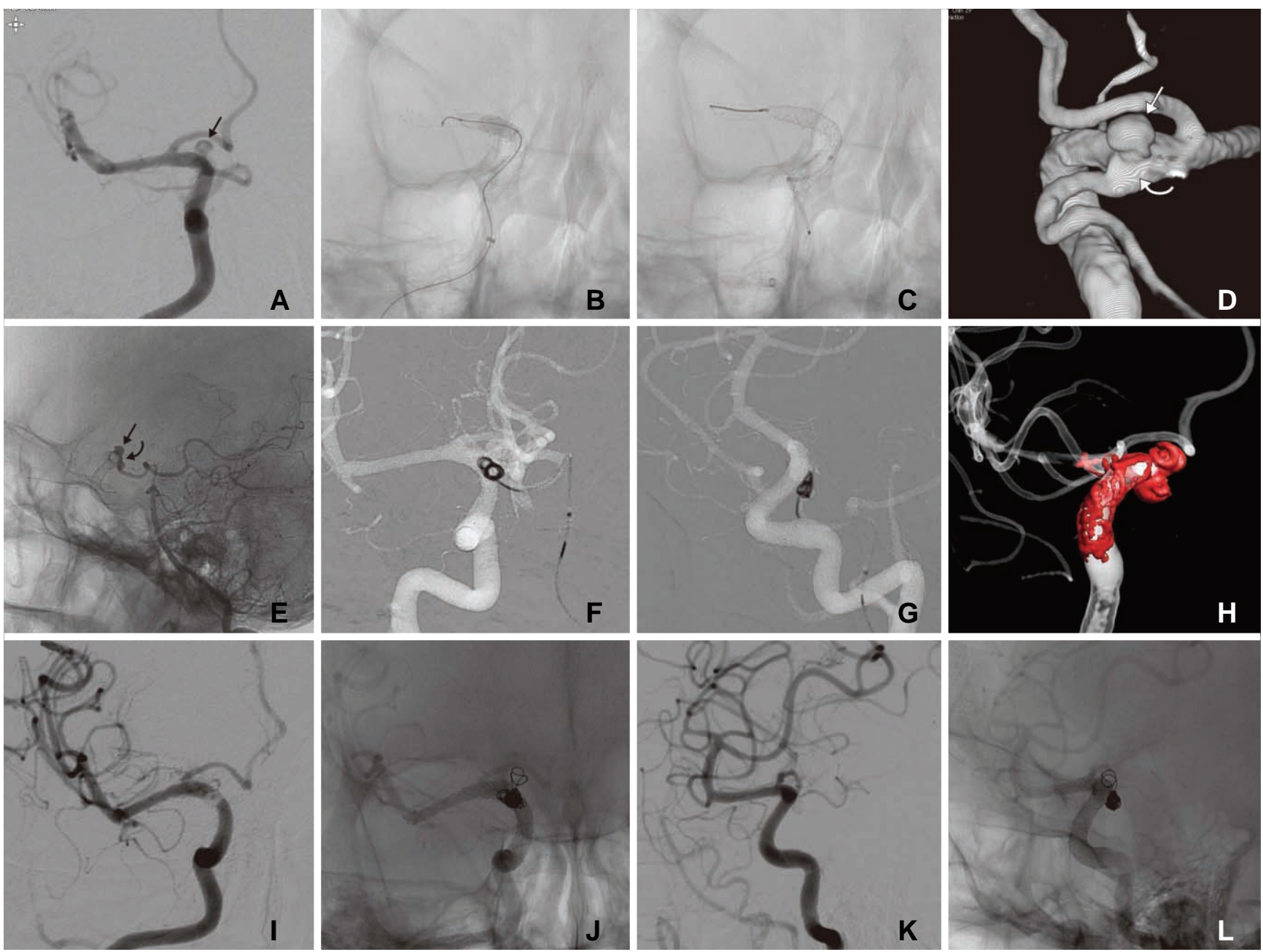

Fig. 3. Follow-up DSA and additional treatment in our institute (A) Follow-up DSA 10 days after the first PED deployment revealed intraaneurysmal contrast filling (arrow) and the reopening of the flow of ACA and PCoA. (B) Firstly, balloon angioplasty within the first PED was performed to achieve complete apposition to the ICA wall. (C) Then, one more PED was implanted within the previous PED for stronger flow diversion (D) However, immediate DSA still showed contrast filling into the BBA (arrow). This could be possibly explained by the inflow from the ICA to the fetal-type PCoA, which was connected to the neck of the BBA (curved arrow). (E) Especially, Allcock test showed the connection between BBA and PCoA well (arrow: BBA, curved arrow: the connection between BBA and PCoA). (F, G, and $\mathbf{H}$ ) Eventually, coil trapping at the origin of the fetal-type PCoA was attempted through the PCoA. (I, J, K, and L) Final DSA demonstrated complete occlusion of the origin of the PCoA and no contrast filling into the BBA.

increased [7]. Another explanation is the "inflammatory theory", where it is hypothesized that formation of an instable red thrombus after abrupt blood stagnation in the aneurysm and a high concentration of lytic enzymes generated by captured leukocytes together with the well-known inflammatory perianeurysmal changes may destabilize the aneurysmal wall [8].

The present case may provide an "anatomical theory" for regrowth and rebleeding after FDSs employment. If a BBA is close to a fetal-type PCoA with high blood flow, and any borders of the BBA are connected to the origin of the fetal-type PCoA, a portion of blood flow into the fetal-type PCoA may result in continuous inflow from ICA into the BBA and then into PCoA, which thereby prohibit effective flow diversion. In this circumstance, coil trapping of the origin of the fetaltype PCoA after FDS employment can be considered to disconnect continuous blood flow for successful flow diversion to bypass the BBA. Recently, Tsang et al also reported that unruptured PCoA aneurysms with persistent fetal-type circulation appears to be particularly refractory to flow divert treatment, especially when the aneurysm incorporates a significant portion of the PCoA, and suggested that alternative or adjuvant modalities should be considered for this subgroup [9].

It is difficult to conclude our hypothesis is valid with only one case. Furthermore, there was a complicating factor, as three self-expandable stents were placed prior 


\section{Ki-Su Park, et al.}

to placement of the PED, which may be associated with an increased risk of failure for PED deployment. However, our case showed the PEDs spanned enough of the length of the existing stents, especially on the proximal end. Therefore, it is possible that this specific circumstance regarding dominant PCoA proximity, such as the present case, can be important to FDS treatment, even with unruptured aneurysms.

\section{CONCLUSIONS}

The PED can be considered as a rescue treatment for ruptured BBAs less amenable to more established techniques of treatment. This case suggests the clinical significance of the fetal-type PCoA. When a BBA is close to the origin of the fetal-type PCoA, it may be important to determine if there is a connection between the fetal-type PCoA and the BBA, and if a connection is present, additional coil trapping to occlude the connection may achieve effective flow diversion and prevent regrowth and rebleeding.

\section{Acknowledgments}

We also appreciate Wade Martin of Emareye for his critical English revision.

\section{References}

1. Park J. Blood blister-like aneurysm with rupture point close to origin of anterior choroidal artery. J Korean Neurosurg Soc 2014;56:500-503

2. Nerva JD, Morton RP, Levitt MR, Osbun JW, Ferreira MJ, Ghodke BV, et al. Pipeline Embolization Device as primary treatment for blister aneurysms and iatrogenic pseudoaneurysms of the internal carotid artery. J Neurointerv Surg 2015;7:210-216

3. Nakagawa F, Kobayashi S, Takemae T, Sugita K. Aneurysms protruding from the dorsal wall of the internal carotid artery. $J$ Neurosurg 1986;65:303-308

4. Kojima M, Irie K, Fukuda T, Arai F, Hirose Y, Negoro M. The study of flow diversion effects on aneurysm using multiple enterprise stents and two flow diverters. Asian J Neurosurg 2012;7:159-165

5. McAuliffe W, Wenderoth JD. Immediate and midterm results following treatment of recently ruptured intracranial aneurysms with the Pipeline embolization device. AJNR Am J Neuroradiol 2012;33:487-493

6. Cruz JP, O'Kelly C, Kelly M, Wong JH, Alshaya W, Martin A, et al. Pipeline embolization device in aneurysmal subarachnoid hemorrhage. AJNR Am J Neuroradiol 2013;34:271-276

7. Cebral JR, Mut F, Raschi M, Scrivano E, Ceratto R, Lylyk P, et al. Aneurysm rupture following treatment with flow-diverting stents: computational hemodynamics analysis of treatment. AJNR Am J Neuroradiol 2011;32:27-33

8. Turowski B, Macht S, Kulcsar Z, Hanggi D, Stummer W. Early fatal hemorrhage after endovascular cerebral aneurysm treatment with a flow diverter (SILK-Stent): do we need to rethink our concepts? Neuroradiology 2011;53:37-41

9. Tsang AC, Fung AM, Tsang FC, Leung GK, Lee R, Lui WM. Failure of flow diverter treatment of intracranial aneurysms related to the fetal-type posterior communicating artery. Neurointervention 2015;10:60-66 